\title{
Coral reefs: threats and future focusing in over-fishing, aquaculture, and educational programs
}

\author{
L. Molina Domínguez ${ }^{1}$, F. Otero Ferrer ${ }^{2} \&$ M. Izquierdo López ${ }^{1}$ \\ ${ }^{1}$ Grupo de Investigación en Acuicultura, \\ Instituto Canario de Ciencias Marinas, \\ Universidad de Las Palmas de Gran Canaria, Spain \\ ${ }^{2}$ Musée Océanographique de Monaco, Monaco
}

\begin{abstract}
Coral reefs cover less than $0.25 \%$ of the marine environment; however their importance is not only justified by their beauty but also because coral reefs provide food and livelihood to millions of people located in communities around the world especially in developing countries. They are considered the "rainforest of the sea" (Spalding et al., 2001) because of their high productivity and specially their biodiversity. In fact, coral reefs support approximately 4000 species of fish, about 800 of reef-building corals and more than a thousand other invertebrates and sponges. Besides, a great number of species use these ecosystems as reproduction or husbandry areas. $20 \%$ of the world's coral reefs have been effectively destroyed and show no immediate prospects of recovery (Wilkinson 2004). The global threats to coral reefs began to raise alarm after the Rio World Environmental Summit in 1992 and the $7^{\text {th }}$ Symposium of Coral Reefs, starting the first calls in order to stop the decline and to provide effective protection for these ecosystems. The threats affecting coral reefs are great and the degree of damage will depend on the different areas of coral reefs locations. The threats can be classified into three groups (note that two are caused directly or indirectly by human activity):

- Natural disasters, such as tropical storms, inundations, earthquakes, volcanoes, etc. To a great extent coral reefs are generally considered to have a strong potential to recover.

- Global climate change, such as increments of sea surfaces temperature, rising $\mathrm{CO}_{2}$ levels and other pollutants.

- Direct human pressure, such as over-fishing, not only for food consumption but also for aquariology purposes, development of coastal zones (urban, industrial, tourism, and transport coral reefs uses).

Against these threats some possible solutions can and must be developed. This paper focuses on over-fishing and the relationship between aquariology and aquaculture.

Keywords: coral reef, aquariology, aquaculture.
\end{abstract}




\section{Introduction}

Although reefs cover less than one quarter of $1 \%$ of the marine environment, they are considered "the rainforest of the seas" [2] because of their biodiversity, and they are considered to be the most biologically and productive rich ecosystems throughout the world. Coral reefs support over 4000 species of fish (which represent one third of the total marine fish species), about 800 species of coral [3] and a great number of other invertebrates and sponges. Coral reefs host an extraordinary variety of marine plants and animals and it has been estimated so far that only about $10 \%$ of these species have been described by scientists.

Reefs support $39 \%$ of the world's population living within $100 \mathrm{~km}$ of coast. They provide important resources for hundreds of millions of people through both local sustenance and commerce: food, tourism revenue, coastal protection and new medications. For example, AZT (a drug for people with HIV infections) is based on chemicals extracted from a Caribbean reef sponge and more than half of all new cancer drugs are based on marine organisms.

Within these activities, collecting marine ornamental organisms provides one of the few potentially sustainable local industries in many coastal communities with limited resources, and few other options for generating income. At stake is the employment of thousands of people, especially in source nations, and the high incentives for coral reef stewardship which the marine aquarium trade is capable of providing.

Worldwide, coral reefs face anthropogenic damage and many are affected by the same economic activities they support [4]. They face a diversity of risks, such as pollution, sedimentation, bleaching, global warming, tourism, and overfishing not only for ornamental purposes, and also tsunamis in the last years. Each of these pressures is bad enough in itself, but together the cocktail has become lethal. Besides, human-perturbed reefs show a prolonged failure to return to their former state compared with perturbed reefs by natural disturbances [5].

Between these threats ornamental fisheries should not be forgotten. The marine ornamental trade began as long as ago the 1930s, and it has now expanded into a multi-million dollar industry, between 28 and 44 millions dollar annually and a global annual catch of about 14 million to over 30 million fish [6], although these figures should be considered with caution due to the lack of contrasted data. .

It must be underlined that nearly all tropical marine fish and invertebrates are taken from the wild coral reefs and adjacent habitats. On the other hand, severe decrease or local extinction of reef organisms might cause, directly or indirectly, deterioration in coral abundance [5]. Reefs of Southeast Asia are the most important source of the aquarium trade, and because of that they are particularly at risk [7] taking into account that damaging techniques of fishing are sometimes used and target species over-exploited in these areas.

Unlike freshwater aquaria species, where $90 \%$ of species are currently farmed, the great majority of marine aquaria are stocked form wild caught species [8]. Aquaculture has experienced a great development of culture 
technologies in the last decades, related to the increase of the world production. All these technologic improvements could be applied to the reproduction and larval culture of species with ornamental interest, with a market already established. Overexploitation of some species fisheries could be diminished, and their is the possibility of repopulation of natural ecosystems in some highly affected cases [9].

\section{An overview of present status of coral reef}

The World Atlas of Coral Reefs prepared by the United Nations Environment Programme World Conservation Monitoring Centre (UNEP-WCMC) provides a new global estimate for coral reefs world-wide: $284300 \mathrm{sq} \mathrm{km}$, an area just half the size of France. For the first time, it also provides reef area estimates for individual countries (Fig.1.) and includes detailed maps and statistics for all the world's coral reef nations.

Indonesia, followed by Australia and Philippines are the largest reef nations, while France comes in fourth $\left(14,280 \mathrm{~km}^{2}\right)$, United Kingdom is $12^{\text {th }}\left(5500 \mathrm{~km}^{2}\right)$, and United States $16^{\text {th }}\left(3770 \mathrm{~km}^{2}\right)$, most located in overseas territories.

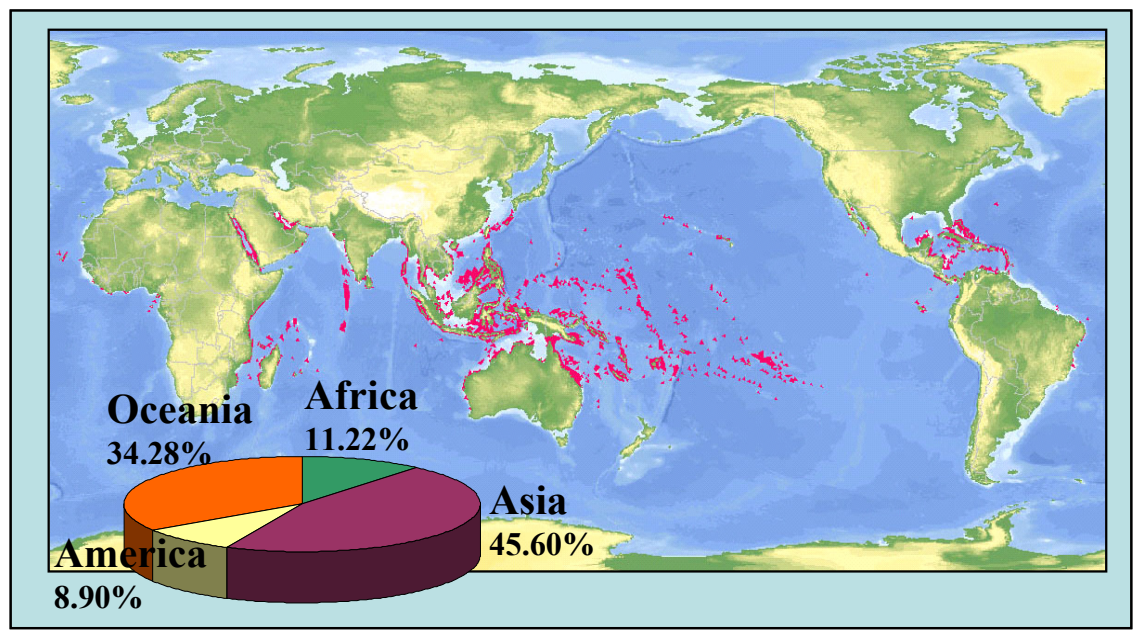

Figure 1: Coral reefs of the world (adapted from www.reefbase.org).

The threats to coral reef and their associated ecosystems vary widely between countries. Most of them are related to the lack of sustainable income-generating opportunities and it must be addressed to achieve sustainable conservation of coral reefs.

Nearly $60 \%$ of the earth's coral reefs are threatened by human activity, they are among the least monitored and protected natural habitats in the world (Fig. 2). 




Figure 2: Percent of affected coral reefs within the different continents referred to the global contribution of each one. Solid colours: unaffected area; soft colours: affected area.

- Coral reefs of Southeast Asia, the most species-rich on earth, are the most threatened of any region. More than $80 \%$ are at risk, primarily from coastal development and fishing-related pressures.

- Nearly two-thirds of Caribbean reefs are in jeopardy. Most of the reefs on the Antilles chain, including the islands of Jamaica, Barbados, Dominica and other vacation favourites, are at high risk. Reefs off Jamaica, for example, have been ravaged as a result of overfishing and pollution.

- Approximately $30 \%$ of East African reefs are at risk. Although levels of estimated reef destruction range widely within the region. Coastal population and coral bleaching are the two primary threats.

- Most United States reefs are threatened. Almost all the reefs off the Florida coast are at risk from a range of factors, including run off of fertilizers and pollutants from farms and coastal development. Close to half of Hawaii's reefs are threatened, while virtually all of Puerto Rico's reefs are at risk.

- Most pacific coral reefs are healthy, less than $10 \%$ are described as threatened, because of effective management or because they are remote from the continent and have low numbers of inhabitants. Generally, there was more damage to the coral reefs through natural events such as cyclones and coral bleaching, than by direct human activities, although climate changes could have an effect.

\section{Conservation measures}

A number of measures can be taken to reduce the threats affecting coral reefs. Focusing in ornamental industry, the lack of contrasted data is one of the main 
problems in order to conserve the population of ornamental organisms living in coral reefs. An effective way of monitoring the fisheries is as simple as to collect data on catch by collectors, including fish that die during the catch. These are in use in Australia, Palau, Cook Islands, Maldives Islands, and Sri Lanka.

Marine ornamental fisheries need to be managed in such a way that they are biologically sustainable and do not conflict with other resource uses. The aquarium trade if managed properly could support long-term conservation and sustainable use of coral reefs. In order to achieve this objective some arguments must be emphasized.

\subsection{Developing sustainable fishing and husbandry practices}

Destructive fishing techniques include the use of sodium cyanide and other chemicals, to stun and catch fish. Estimation of its use is difficult to obtain due to the clandestine nature of these practices. Other collection techniques such as dynamite and explosives are also commonly used for food fishing. During the coral collection for trade, many more colonies may be damaged or broken. All these methods cause terrible damage to organism populations but also to the reef habitat itself. Obviously, these practices must be overcome; some other collection methods have minimal impacts on coral reef. Cyanide fishing is illegal in most countries, but more efforts in producer countries are needed: changing their policies, promoting awareness campaigns, regulating the importation, distribution and use of the cyanide. It is necessary to limit the collecting effort by controlling the number of collectors or establishing quotas on numbers of fish captured or exported [3] based on scientific research carried out in order to decide the appropriate numbers. Consumers also have an important role to play: if the demand of sustainable fishing organisms increases it is likely that this will affect producer countries. One of the best examples is Marine Aquarium Council (MAC) Certification launched in late 2001 that provides aquarists with the only comprehensive "reef to retail" system, a tool to identify marine aquarium organisms that originate from managed reefs, are caught using non-destructive methods, and are handled and transported according to best practices. An increasing number of collectors, wholesalers and retailers in Asia, the Pacific, North America and Europe are now MAC certificated.

\subsection{Promoting the implantation of locally based projects of ornamentals cultivation}

This action is very important in order to sustain the trade and to present an alternative livelihood to local people. Mariculture facilities must be designed in order to create job opportunities for coastal populations, especially fishermen. Some initiatives are already working (i.e. Lombok Frags, Indonesia) and it could be extended. In this way a new basis of subsistence may reduce the anthropogenic pressure and increase awareness protection in their reef areas. However, the intensive use of net penned fish could affect corals, although there are some discrepancies about this subject [5, 10-12]. 


\subsection{Promoting the research and the culture of ornamental species}

The limited number of fish species bred in captivity can be pointed out. Due to the increasing regulations or restrictions about the trade of some species, without aquaculture to provide specimens for exhibits, many species may not be accessible for display and for exchange with other institutions [13]. Moreover, the knowledge and technology of establishing and maintaining the live coral reefs exhibits is important for the conservation and the restoration of the wild coral reefs. Besides, as controllable facilities, they are powerful tools for the research of global environment change on coral, both at the community and the organism levels [14]. Public aquariums have a great opportunity for future conservation and that also means a great responsibility.

\subsection{Coral reef conservation and restoration}

Coral reef conservation focuses on "passive" measures in order to allow the ecosystem to recover with minor human actions. Coral reef restoration is based on artificial manipulations in order to improve the coral state. Various methodologies for coral restoration have been proposed [15], such as coral culture, chemical products, and artificial reefs. Farmed coral could be used to restore damaged reef areas and aquarium trade or the pharmaceutical industry. Not only asexual but also sexual reproduction must be used in order to preserve genetic diversity [16]. An artificial reef is a method not used specifically for this purpose, but with interesting potential at least in boosting the availability of species. It must be pointed out that it must be a temporary solution with the possibility of the generation of new problems [17]. Because of that, a great effort to improve this tool must be made, for example by specific design. Artificial reefs must be carefully sited and sized in order to provide abundance and diversity of specimens. More research will be necessary to promote the improvement of these techniques.

\subsection{Domestic or public aquaria to educate conservation}

Traditionally public aquarium's tasks are to communicate, instruct and educate people on aquatic issues, but today's aquaria could have an important role in applied or basic scientific research associated with other institutions, providing scientific publications, connecting different stakeholders (experts, politicians, industry, non-governmental associations, and public in general), and creating exhibits in order to show environmental concerns. New modern aquarium must play an important role in conservation and education programs and those also related to the research projects. As our planet becomes more threatened by social impact, the main objectives of an educational promotion at a public aquarium must be to engage visitors to pollute less, support wise political practices in conservation (catch limits, certification, better identification of fishing areas etc), and engage the next generation in attitudinal readiness. By making these changes, ecosystems that have been threatened because of bad practices can be allowed to recover [18]. Moreover, live coral reef exhibits are one of the most 
powerful tools to hold people's attention and provide an understanding of the beauty, uniqueness and importance of coral reefs. The popularity of these exhibits could be used to raise public awareness.

\section{Conclusions}

The most important actions for promoting healthy coral reef ecosystems depend largely on the efforts by local governments, community groups, environmental organizations and the private sector. More accurate trade data and more information about target species are needed to establish regulations, marine reserves, quotas and maximum yield productions, in order to promote the ornamental trade as a sustainable basis. Finally, further research into ornamental species technologies in order to diminish the pressure off wild stocks and increase the effectiveness of aquaculture facilities. Some of these projects should be locally based in source countries as an alternative livelihood for fishing communities, integrating conservation and progress.

\section{References}

[1] Status of coral reefs of the world. Australian Government. Australian Institute of Marine Science. Online. http:/www.aims.gov.au/pages/ research/coral-bleaching/scr2004/.

[2] Spalding, M., Ravilious, C. and Green, E. World Atlas of coral reefs. University of California Press, Berkeley, USA.pp.11-13, 2001.

[3] Paulay, G. Diversity and distribution of reef organisms. Life and Death of coral reefs, ed. C. Birkeland, Chapman and Hall, New York, USA. pp. 298-353. 1997.

[4] Wielgus, J., Chadwick-Furman, N., Dubinsky, Z. Shechter, M. \& Zeitouni, N. Coral reefs 21, pp.253-259.2002.

[5] Loya, Y. The coral reefs of Eilat -Past, Present and Future: Three decades of coral community structure studies. Coral health and disease. Springer, pp. 1-34. 2004.

[6] Wood, E. Global advance in conservation and management of marine ornamental resources. Aquarium Sciences and Conservation, 3 (1-3), pp. 65-7. 2001.

[7] Burke L., Selig, E. \& Spalding, M. Reefs at risk in Southeast Asia. World Resources Institute, Washington DC, USA, 2002.

[8] Andrews, C. The ornamental fish trade and fish conservation. Journal of fish biology, 37 (Supplement A), pp. 53-59, 1990.

[9] Molina Domínguez, L. \& Ounaïs, N. The sustainability of Aquarium trade. Proc. of XI World Aquaculture International Congress, Nusa Dua, Bali (Indonesia) p. 53. 2005.

[10] Bongiorni, L., Shafir, S. \& Rinkevich, D. Effects of particulate matter released by a fish farm (Eilat, Red Sea) on survival and growth of Stylofora pistillata coral nubbins. Marine Pollution Bulletin, 46, pp. 1120 1124. 2003. 
[11] Rinkevich, B. Nutrient enrichment and coral reproduction: between truth and repose (a critique of Loya et al.). Marine Pollution Bulletin, 50, pp.111-113. 2005.

[12] Rinkevich, B. What do we know about Eilat (Red Sea) reef degradation? A critical examination of the published literature. Journal of Experimental Biology and Ecology, 327, pp. 183-200. 2005.

[13] Page, G. Gomezjurado, J. \& Ashley, A. Ecological Aquaculture a new paradigm for conservation. Proc. of the VI International Aquarium Congress, Monterey, California, USA, p. 53. 2004.

[14] Fan, T., Kuo, F. \& Fang, L. The coral reef Mesocosms in the national Museum of Marine Biology and Aquarium, Taiwan. Proc. of the VI International Aquarium Congress, Monterey, California, USA, p. 31.2004.

[15] Rinkevich, B. Conservation of coral reefs through active restoration measures: recent approaches and last decade progress. Environmental Science \& Technology, 39, pp. 4333-4342. 2005.

[16] Petersen, D., Laterveer, M. \& Schuhmacher, H. Methods to enhance sexual recruitment for restoration of damaged reefs. Marine Biology, 146, pp. 937-942. 2005.

[17] McAllister, D.E. Is mariculture the remedy to problems of coral reefs of coastal communities? SPC Live Reef Information Bulletin 5 pp. 47-48, 1999.

[18] Sonnenschein, L. Aquariums reaching the public with Conservation Campaigns. Proc. of the VI International Aquarium Congress, Monterey, California, USA, p. 53. 2004. 